\title{
Estudo das relações entre compreensão em leitura e desempenho acadêmico na universidade
}

\author{
Katya Luciane de Oliveira \\ Acácia Aparecida Angeli dos Santos \\ Ricardo Primi \\ Universidade São Francisco
}

\begin{abstract}
Resumo
Este estudo objetivou explorar as relações entre compreensão em leitura e desempenho acadêmico em universitários das áreas de ciências humanas, exatas e biológicas. A amostra foi composta por 412 alunos dos cursos de letras, psicologia, matemática e odontologia. Os escores obtidos no teste de Cloze foram correlacionados com o desempenho médio nas disciplinas cursadas pelos alunos no primeiro ano do curso. Foi utilizado um texto preparado segundo a técnica de Cloze e a coleta de dados ocorreu de forma coletiva. Em todos os cursos os escores do Cloze se correlacionaram ao desempenho acadêmico, à exceção do curso de matemática. Os resultados indicaram a existência de uma associação significativa entre compreensão em leitura e desempenho acadêmico.

Palavras-chave: compreensão em leitura; rendimento acadêmico; universitários.
\end{abstract}

\begin{abstract}
Relations study between reading comprehension and academic performance in the university This research was meant to explore the relation between reading comprehension and academic performance in freshman students from engineering, social sciences and biological areas The Cloze's score were correlated with the average performance in each course attended by first year students. The sample was composed of 412 students in language and literature, psychology, mathematics and dentistry courses. A text prepared, in accordance with Cloze's Technique, was answered by the students collectively. In all courses, but mathematics, a significant association between reading comprehension and academic performance was found.

Keywords: reading comprehension; academic performance; freshman students.
\end{abstract}

A leitura é uma habilidade para a qual há a necessidade de um aprendizado contínuo, pois, quanto mais o homem toma conhecimento de seu mundo e de novas palavras, mais apto ele será no reconhecimento delas. Ler é muito mais do que interpretar as letras para compreender a mensagem, visto que inclui o sentimento e a atribuição de significado ao texto, além do relacionamento do conteúdo com outros conhecimentos já adquiridos.

A leitura expressa um grande passo para o homem em sua busca do conhecimento, proporcionando-lhe a capacidade de refletir e opinar sobre diversos aspectos da vida. Outro aspecto a ser enfatizado é o papel social da leitura, entendida como um produto cultural, sabendo-se que num mundo onde a informação disponível é cada vez maior, as pessoas não instrumentalizadas para acessá-las serão excluídas social e culturalmente (Drouet, 1995; Ellis, 1995; Gregoire \& Piérart, 1997).

O reconhecimento da importância da leitura na vida do homem é evidente, visto proporcionar reflexões e questionamentos acerca dos fatos, estando presente em todos os momentos do cotidiano. Independentemente da explicação de como se processa o de- senvolvimento da habilidade da leitura, é sabido que ela representa um grande passo para a aquisição do conhecimento. É por meio dela que se adquire uma percepção singular do mundo. A leitura oferece também uma contribuição para o funcionamento e desenvolvimento do pensamento crítico, levando o leitor a questionar e avaliar o texto lido, dentro de um referencial próprio de seus conhecimentos, conceitos e valores (Santos, 1990a; Witter, 1997).

Como ressalta Perfetti (1992) a compreensão em leitura ocorre quando há um entendimento das sentenças do texto, que se constitui de um conjunto de sentenças relacionadas, que convergem para uma finalidade de idéias nele explicitadas. Todavia, a compreensão verdadeira exige muito mais do que a decodificação das sentenças, considerando que o leitor acrescenta os seus conhecimentos e experiências anteriores, baseada na sua percepção sobre o mundo. Espera-se que o leitor fluente adquira uma compreensão crítica do texto, avalie a idéia do autor e possa tirar sua própria conclusão sobre o tema.

Duran (1981) e Cardoso (1994) afirmam que a conclusão da educação superior não é sinônimo de 
oportunidades de empregos bem remunerados, apenas em função da qualificação recebida pelo aluno durante o curso realizado. É sabido que grande parte desses alunos chegam ao final do curso com sérias deficiências no conhecimento, resultado de uma baixa habilidade em leitura e, em especial, a incapacidade de abstrair as idéias mais importantes de um texto, necessárias para seu conhecimento e qualificação no mercado profissional.

Considerando esse contexto, é dever da universidade proporcionar ao estudante uma formação que lhe propicie condições de possuir domínio das habilidades envolvidas na leitura, principalmente no que concerne à leitura técnico-científica, fundamental ao futuro desempenho profissional desse estudante. A compreensão em leitura, essencial para o sucesso no ensino superior, está associado à maturidade em leitura, que pode ser implementada por meio de programas específicos de intervenção. Assim, o papel da universidade é planejar, desenvolver e administrar programas para a superação das limitações relacionadas às eventuais dificuldades detectadas (Santos, 1990a; Witter, 1997).

Pesquisas sobre as dificuldades de leitura apresentadas pelos universitários têm corroborado com essas afirmações. Assim, em um estudo comparativo sobre desempenho em leitura em estudantes universitários dos cursos de engenharia e fonoaudiologia, Oliveira (1996) evidenciou que os participantes não apresentavam o desempenho em leitura esperado para leitores do ensino superior. Ao pesquisarem as condições de estudo de universitários, Mercuri (1992) e Carelli (1996) contataram que esses estudantes apresentam dificuldades quanto à compreensão e redação de textos No mesmo sentido, Egypto (1983) e Pellegrini (1996) evidenciaram que os estudantes possuem dificuldades de abstrair do texto as idéias relevantes e apresentam um nível de compreensão aquém do esperado para estudantes nesse estágio de escolaridade. Verifica-se, ainda, que muitos alunos chegam ao ensino superior com deficiências em leitura por não haver, nos níveis escolares anteriores, ações que garantam os mecanismos necessários para o diagnóstico e enfrentamento dessa situação.

Assim, a importância do diagnóstico do perfil dos ingressantes torna-se fundamental para que suas habilidades e competências sejam adequadamente avaliadas e para que lhes sejam oferecidas, ao longo do curso superior, atividades preventivas e remediativas que possibilitem não só o necessário desenvolvimento cognitivo, como também o desejável desenvolvimento pessoal (Santos, Primi, Taxa \& Vendramini, 2002; Santos, Vendramini, Suehiro \& Santos, 2002). Nesse sentido, Witter (1997) defende que primeiramente é necessário avaliar o desempenho em leitura nos universitários para posteriormente encaminhá-los a programas específicos em função da deficiência detectada.

Desse modo, ressalta-se que o teste de Cloze é um instrumento que vem sendo muito utilizado para diagnosticar as dificuldades de compreensão de leitura nos diversos níveis de escolaridade. Sua alta aceitação tem sido demonstrada pelas inúmeras publicações discorrendo sobre as diversas possibilidades de utilização desse recurso (Santos, 1990a; Bedento \& Moreira, 1990; Bensoussan, 1990; Kletzien, 1991; Abraham \& Chapelle, 1992; Storey, 1997; Vicentelli, 1999).

Santos (1991) afirma que o teste de Cloze foi criado em 1953 por Taylor e constitui-se de um texto com duzentas palavras, aproximadamente, do qual se omitem todos os quintos vocábulos, que são substituídos por um traço do tamanho da palavra omitida. O leitor deverá completar o texto com a palavra que acreditar ser mais apropriada. Garrido (1988) observa que a técnica de Cloze é um instrumento estruturado, simples e válido, quando o objetivo é avaliar o nível de compreensão de leitura. Tem sido utilizado em diversos níveis do ensino formal devido ao seu grau de confiabilidade, sendo que atualmente há evidências de validade do Cloze, tanto como medida de inteligibilidade do texto (Molina, 1979; Mello, 1986), quanto como indicador da habilidade em compreensão de leitura (Bitar, 1989; Santos, 1991, Santos e cols., 2002).

Anastasi e Urbina (2000) expõem que os escores de um teste são interpretados com referência aos resultados obtidos por um grupo representativo. Dessa forma, o escore bruto de um determinado sujeito é analisado com base na amostra de padronização. Os escores indicam a posição do sujeito na amostra normativa e com isso há a possibilidade de predizer qual será o desempenho do sujeito em referência a outros indivíduos. $\mathrm{O}$ escore em um teste diz respeito à interpretação atribuída aos diferentes níveis de desempenho. Com base nesse pressuposto, Santos e cols. (2002) investigaram as propriedades psicométricas do teste de Cloze para a avaliação da compreensão da leitura. Valendo-se da teoria de resposta ao item e das análises dos escores do Cloze de 612 universitários, demonstraram que o instrumento pode ser utilizado para a verificação do nível de compreensão em leitura.

Estudos anteriores têm demonstrado a relação existente entre a compreensão em leitura, hábitos de estudo e desempenho acadêmico e a validade da técnica de Cloze como remediativa da compreensão em leitura com universitários (Santos (1990a; 1991). Utilizando ainda o procedimento de Cloze para o diagnóstico e para o desenvolvimento da compreensão em leitura, Santos (1997) elaborou um programa de remediação de leitura, voltado a ingressantes que apresentavam dificuldades em compreensão e verificou a possibilidade de sua para o desenvolvimento de um leitor mais maduro, como é desejável nessa etapa de escolaridade.

Desse modo, constata-se-se que, mesmo no ensino superior, há possibilidade de se atuar na remediação do baixo nível de compreensão em leitura ainda que o vestibular tenha permitido a entrada daqueles alunos que não estavam suficientemente preparados. Vale ressaltar que esses universitários, ao final de sua formação, devem possuir pensamento crítico desenvolvido e maior conhecimento sobre si mesmos, de forma a possuírem condições de tomar decisões. A 
leitura é um dos canais de acesso a esses comportamentos, e a instituição de ensino superior deve estar preocupada em avaliar o nível de habilidade que o aluno possui, para poder proporcionar, no âmbito das várias disciplinas, a oportunidade de superação de eventuais limitações.

Assim, o presente trabalho teve como objetivo verificar se o nível de compreensão em leitura está relacionado com o desempenho acadêmico em disciplinas de cursos das áreas de ciências exatas, humanas e biológicas.

\section{MÉTODO}

\section{Sujeitos}

Nesta pesquisa utilizou-se os resultados do Teste de Cloze e as notas escolares das disciplinas específicas dos primeiros anos de 412 universitários ingressantes dos cursos de letras $(12,37 \%$; $N=51)$, psicologia (42,71\%; $N=176)$, matemática $(7,52 \%$; $N=31)$ e odontologia $(37,37 \% ; N=154)$.

No curso de letras 92,2\% ( $\mathrm{N}=47)$ dos estudantes representavam o sexo feminino e 7,8\% (N=4) o masculino. No que se refere ao período, todos os alunos estudavam no período noturno. A média de idade foi de 24 anos e 5 meses $(D P=7,6)$.

O curso de psicologia contava com 92\% (N=162) de estudantes do sexo feminino e $8 \%(N=14)$ do sexo masculino. Quanto ao período, 29\% (N=51) eram do diurno e $71 \%(N=125)$ do noturno. A média de idade foi de 22 anos e 7 meses $(D P=5,7)$.

A porcentagem de estudantes do sexo feminino no curso de matemática foi de $77,41 \% \quad(N=24)$ e do masculino $22,5 \%(N=7)$. Todos os alunos estudavam no período noturno e a média de idade foi de 22 anos e 5 meses $(D P=4,5)$.

$\mathrm{O}$ curso de odontologia apresentou uma porcentagem de $68,2 \%(N=105)$ de estudantes do sexo feminino e $31,8 \%(N=49)$ do sexo masculino, sendo que $55,84 \%(N=86)$ estudavam no período diurno e $44,15(N=68)$ no noturno. A média de idade foi de 19 anos e 8 meses $(D P=2,3)$.

\section{Instrumento}

Teste de Cloze - foi utilizado um texto adaptado de Luís Fernando Veríssimo (1995) intitulado 'Desentendimento'. O texto continha aproximadamente 250 vocábulos, do qual se omitiu sempre o quinto vocábulo, sendo que o espaço deixado foi proporcional ao tamanho da palavra omitida.

\section{Critérios de Avaliação}

Notas escolares: médias relativas ao primeiro semestre, obtidas na secretaria da instituição, de todos os conteúdos específicos, dos cursos de letras, psicologia, matemática e odontologia.

Os conteúdos específicos analisados no curso de letras foram conversação em inglês I, estrutura e funcionamento do ensino fundamental e médio, filosofia, laboratório de língua inglesa I, língua inglesa I, língua portuguesa I, língua/literatura latina, lingüística I, leitura e produção de textos I, psicologia da educação e teoria da literatura.

As disciplinas analisadas no curso de psicologia foram antropologia, biologia, estatística, estrutura e funcionamento do ensino médio, filosofia I, história da psicologia, introdução a formação profissional, leitura e produção de textos, metodologia científica, psicologia desenvolvimento I, psicologia geral e técnicas de exames psicológicos.

No curso de matemática os conteúdos trabalhados foram cálculo e geometria analítica I, contabilidade geral, filosofia, física geral e experimental I, informática básica, matemática comercial/financeira e português instrumental.

Anatomia, biologia, bioquímica, ciências sociais, escultura dental, histologia/embriologia, metodologia científica e micro/imunologia e parasitologia foram as disciplinas analisadas no curso de odontologia.

Número de acertos obtidos no teste de Cloze, cuja forma de correção foi literal (aceita como correta a palavra exata que foi omitida), tal como proposto e fundamentado por Bitar (1989).

\section{Procedimento}

As provas foram aplicadas em uma sessão, em horário de aula previamente cedido pelo professor, aos sujeitos que consentiram em participar da pesquisa.

\section{RESULTADOS}

Os escores no teste de Cloze foram correlacionados com o desempenho médio nas disciplinas cursadas pelos alunos do primeiro ano dos cursos de letras, psicologia, matemática e odontologia. Das 38 disciplinas analisadas 52,63\% ( $N=20)$ apresentaram uma relação estatisticamente significativa com a compreensão em leitura. Entretanto, um número razoável de disciplinas $(47,36 \% ; N=18)$ não obteve relação significativa com os escores do Cloze.

Os conteúdos específicos do curso de letras que apresentaram uma relação estatisticamente significativa com os escores do Cloze são apresentados na Tabela 1.

Tabela 1 - Distribuição das médias, desvios padrões e correlações entre os escores do Cloze e o desempenho acadêmico nos conteúdos específicos do curso de letras $(N=51)$.

\begin{tabular}{|c|c|c|c|c|}
\hline Conteúdos Específicos & $\begin{array}{c}\text { Médias } \\
\text { Escolares }\end{array}$ & $D P$ & $r$ & $p$ \\
\hline Conversação em inglês & 6,40 & 1,60 & 0,472 & 0,002 \\
\hline Filosofia & 7,49 & 1,08 & 0,351 & 0,023 \\
\hline
\end{tabular}




\begin{tabular}{lllll}
\hline Laboratório de língua inglesa I & 7,58 & 1,27 & 0,489 & 0,001 \\
Língua inglesa I & 7,53 & 1,33 & 0,417 & 0,005 \\
Língua portuguesa I & 7,15 & 1,02 & 0,456 & 0,002 \\
Língua/literatura latina & 6,33 & 1,14 & 0,333 & 0,029 \\
Leitura e produção de textos I & 6,10 & 1,32 & 0,295 & 0,058 \\
Teoria da literatura & 5,96 & 1,43 & 0,309 & 0,044 \\
\hline
\end{tabular}

Das onze disciplinas analisadas, oito se relacionaram aos escores do Cloze. As disciplinas que não apresentaram relação significativa com a compreensão em leitura foram lingüística I, psicologia da educação e estrutura e funcionamento do ensino fundamental e médio, ressaltando que essa disciplina obteve um resultado marginalmente significativo $(r=0,290 ; p=0,063)$.

A Tabela 2 mostra os conteúdos específicos do curso de psicologia, que obtiveram uma relação estatisticamente significativa com a compreensão em leitura.

Tabela 2 - Distribuição das médias, desvios padrões e correlações entre os escores do Cloze e o desempenho acadêmico nos conteúdos específicos do curso de psicologia $(N=176)$.

\begin{tabular}{|c|c|c|c|c|}
\hline Conteúdos Específicos & $\begin{array}{l}\text { Médias } \\
\text { Escolares }\end{array}$ & $D P$ & $r$ & $p$ \\
\hline Biologia & 5,94 & 1,10 & 0,275 & 0,000 \\
\hline Estrutura e funcionamento do ensino médio & 6,32 & 0,89 & 0,204 & 0,009 \\
\hline Introdução à formação profissional & 6,63 & 0,99 & 0,246 & 0,002 \\
\hline Leitura e produção de textos & 6,17 & 0,90 & 0,228 & 0,003 \\
\hline Psicologia do desenvolvimento I & 7,44 & 1,09 & 0,262 & 0,001 \\
\hline Técnicas de exame psicológico I & 6,43 & 1,11 & 0,270 & 0,000 \\
\hline
\end{tabular}

Observou-se que, das doze relações estudadas, seis foram estatisticamente significativas. O desempenho acadêmico das disciplinas antropologia, estatística, filosofia I, história da psicologia, metodologia científica, psicologia geral não se correlacionou significativamente com a compreensão em leitura.

No curso de matemática das sete disciplinas analisadas nenhuma apresentou relação estatisticamente significativa com a compreensão em leitura, considerando $p<0,05$. Sendo que a disciplina contabilidade geral apresentou um nível marginalmente de significância $(r=0,333 ; p=0,073)$

$\mathrm{Na}$ Tabela 3 foram elencados os conteúdos específicos do curso de odontologia que obtiveram uma relação estatisticamente significativa com a compreensão em leitura.

Tabela 3 - Distribuição das médias, desvios padrões e correlações entre os escores do Cloze e o desempenho acadêmico nos conteúdos específicos do curso de odontologia $(N=154)$.

\begin{tabular}{|c|c|c|c|c|}
\hline Conteúdos Específicos & $\begin{array}{c}\text { Médias } \\
\text { Escolares }\end{array}$ & $D P$ & $r$ & $p$ \\
\hline Biologia & 5,58 & 0,63 & 0,242 & 0,005 \\
\hline Bioquímica & 4,91 & 0,79 & 0,164 & 0,058 \\
\hline Ciências sociais & 7,37 & 1,10 & 0,288 & 0,001 \\
\hline Histologia/embriologia & 5,67 & 1,38 & 0,270 & 0,002 \\
\hline Metodologia científica & 6,66 & 0,90 & 0,348 & 0,000 \\
\hline Micro/imunologia e parasito & 6,01 & 0,92 & 0,185 & 0,032 \\
\hline
\end{tabular}

Das oito disciplinas estudadas, seis apresentaram relação significativa com a compreensão em leitura. As disciplinas que não apresentaram relação estatisticamente significativa com os escores do Cloze foram escultura dental e anatomia, sendo que essa disciplina apresentou um nível marginalmente de significância $(r=0,154 ; p=0,074)$.

\section{DISCUSSÃO E CONCLUSÃO}

Os resultados, de uma forma geral, evidenciam que há uma associação significativa entre compreensão em leitura e desempenho em conteúdos específicos. Há de se considerar que, além da relação encontrada na maioria das disciplinas, três disciplinas apresentaram relação marginalmente significativa entre as variáveis estudadas. Esse dado demonstra que 60\% dos 
conteúdos específicos requerem habilidade de compreensão em leitura e corrobora com as pesquisas realizadas anteriormente por Santos (1990a, 1991) e Vicentelli, (1999).

Todavia, diferentemente do esperado, um número considerável de disciplinas não apresentou relação com a compreensão em leitura. Estranha-se o fato de que algumas disciplinas que requerem atividade intensa de leitura não tenham apresentado correlação com o desempenho no teste de Cloze, como ocorreu no curso de letras com as disciplinas estrutura e funcionamento do ensino fundamental e médio e psicologia da educação; no curso de psicologia com as disciplinas antropologia, filosofia I, história da psicologia e psicologia geral e no curso de matemática com a disciplina filosofia. No mesmo sentido, Garrido (1988) observa que pesquisas realizadas em universidades americanas também apresentaram índices de correlação baixos quando compararam os escores do teste de Cloze com os resultados de testes normativos de habilidade geral em leitura. Tais conclusões apontam para a necessidade de realização de novas pesquisas sobre o tema, de forma a elucidar as questões aqui levantadas.

Outro aspecto que não pode ser ignorado é que o curso de matemática totalizava sete disciplinas, das quais nenhuma apresentou relação com a compreensão em leitura. Os conteúdos que não se correlacionaram com o Cloze no curso de matemática somaram-se às dos demais cursos, e isso pesou no resultado final (um número considerável de disciplinas não se correlacionou com os escores do Cloze). A falta de relação entre as variáveis no curso de matemática pode ser compreendida, considerandose que a maior parte das disciplinas, com exceção da disciplina filosofia, eram de conteúdos que não exigiam tanto da habilidade de compreensão em leitura para a obtenção de um bom desempenho acadêmico.

Uma análise detalhada das médias dos alunos leva à formulação de algumas hipóteses possíveis para a explicação de tais resultados. A primeira seria a de que a falta de correlação entre a compreensão em leitura e o desempenho acadêmico nessas disciplinas pode estar associado a avaliações de menor exigência. A segunda seria a de que a baixa pontuação no Cloze estaria relacionada à falta de comprometimento do aluno ao responder ao instrumento, visto que os participantes foram recrutados na sala de aula. Como tais aspectos não foram objetos de análise deste estudo sugere-se que outras pesquisas investiguem tais aspectos.

Desejável seria que o desempenho na maioria das disciplinas tivesse se relacionado com a compreensão em leitura. Pois assim, refletiria tanto uma melhor formação técnica do aluno, como o seu desenvolvimento enquanto leitor. Conseqüentemente, isso o auxiliaria a tornar-se um profissional mais competente, visto que, por meio da leitura, adquire-se maior bagagem cultural e sólida formação profissional, como ressaltado por diversos estudos (Santos, 1990a; Cardoso, 1994; Vicentelli, 1999).
Não se pode esquecer, entretanto, as observações oriundas do estudo de Duran (1981) sobre o enorme despreparo dos ingressantes no ensino superior no Brasil, resultado de uma escolarização pobre, associada a diversas desvantagens sociais. Dessa forma, uma questão evidente é que diversos alunos chegam aos cursos superiores sem possuir uma compreensão em leitura satisfatória, e o mais lamentável é que esses alunos muitas vezes saem desses cursos sem possuírem essa habilidade totalmente desenvolvida (Egypto, 1983; Pellegrini, 1996). A compreensão em leitura é um tema de grande importância dentro do contexto universitário, tendo em vista que esse estudante ingressará em um mercado de trabalho competitivo, devendo possuir uma qualificação técnica que lhe será exigida, conforme observam Cardoso (1994) e Witter (1997).

A qualificação, por sua vez, apresenta forte relação com um bom desempenho em leitura. Outro aspecto a ser destacado e questionado é que, se a leitura é tão importante na aquisição do conhecimento como evidenciado por Santos (1990a), Garrido (1988), Oliveira (1996), Witter (1997), entre tantos outros, observa-se que no Brasil esse campo de pesquisa ainda tem muito a ser explorado. Finalmente deve-se lembrar a relevância de meios diagnósticos e de intervenção eficazes para o enfrentamento dessa situação, sendo papel da universidade uma intervenção efetiva para a superação dos problemas relacionados à habilidade de leitura, bem como para o seu desenvolvimento e aprimoramento.

\section{REFERÊNCIAS}

Abraham, R. G. \& Chapelle, C. A. (1992). The meaning of Cloze test scores: an item difficulty perspective. The Modern Language Journal, 76, 468-479.

Anastasi, A. \& Urbina, S. (2000). Testagem Psicológica (7 ed.). Tradução Maria Adriana Veríssimo Veronese. Porto Alegre: Artes Médicas.

Bedento, J. M. \& Moreira, L. C. (1990). Desempenho em leitura entre estudantes de enfermagem: um estudo com a técnica de Cloze. Anais do $2^{\circ}$ Simpósio Brasileiro de Comunicação em Enfermagem. Ribeirão Preto, 701-14.

Bensoussan, M. (1990). Redundancy and the cohesion Cloze. Journal of research in reading, 13 (1), 18-37.

Bitar, M. L. (1989). Eficiência dos instrumentos de avaliação em leitura. Dissertação de Mestrado. Pontifícia Universidade Católica de São Paulo, São Paulo.

Cardoso, S. M. V. (1994). A prática docente no ensino superior particular noturno: um estudo de caso. Tese de Doutorado. Faculdade de Educação da Universidade Estadual de Campinas, Campinas-SP.

Carelli, M. J. G. (1996). Perfil sóciocultural e condições de estudo em alunos da Universidade São Francisco. Dissertação de Mestrado. Faculdade de Filosofia, Ciências e Letras, Universidade São Francisco, Bragança Paulista.

Drouet, R.C. R. (1995). Distúrbios da aprendizagem. São Paulo: Ática. 
Duran, A. P. (1981). Padrões de comunicação oral e compreensão da comunicação escrita na universidade: estudos no Nordeste. Tese de Doutorado. Instituto de Psicologia da Universidade de São Paulo, São Paulo.

Egypto, M. S. R. (1983). Leitura em estudantes universitário: estudo contrastivo de caracterização de nível de desempenho. Dissertação de Mestrado. Universidade Federal da Paraíba, João Pessoa.

Ellis, A. W. (1995). Leitura, escrita e dislexia: uma análise cognitiva (2. ed.). Porto Alegre: Artes Médicas.

Garrido, E. (1988). O ensino da filosofia no $2^{\circ}$ grau e a compreensão de textos: um levantamento em São Paulo e uma aplicação da técnica Cloze. Tese de Doutorado. Faculdade de Educação da Universidade de São Paulo, São Paulo.

Gregoire, J. \& Piérart, B. (1997). Avaliação problemas de leitura: os novos modelos teóricos e suas implicações. Porto Alegre: Artes Médicas.

Kletzien, S. B. (1991). Strategy use by good and poor comprehenders reading expository text of differing levels. Reading Research Quarterly, XXVI (1), 67-86.

Mello, M. T. P. (1986). Estudo psicolingüístico de dois materiais de leitura de História do Brasil, quanto aos aspectos vocabular e figurativo. Tese de Doutorado. Faculdade de Filosofia, Letras e Ciências Humanas, Universidade de São Paulo, São Paulo.

Mercuri, E. (1992). Condições espaciais, materiais, temporais e pessoais para o estudo, segundo depoimento de alunos e professores do curso de graduação da UNICAMP. Tese de Doutorado. Faculdade de Educação da Universidade Estadual de Campinas, Campinas-SP.

Molina, O. (1979). Avaliação da inteligibilidade de livros didáticos de $1^{\circ}$ e $2^{o}$ graus por meio da técnica de Cloze. Tese de Doutorado. Instituto de Psicologia da Universidade de São Paulo, São Paulo.

Oliveira, M. H. M. A. (1996). Funções da leitura para estudantes de graduação. Psicologia Escolar $e$ Educacional, 1 (1), 61-68.

Pellegrini, M. C. K. (1996). Avaliação dos níveis de compreensão e atitudes frente à leitura em universitários. Dissertação de Mestrado. Programa de Estudos Pós-graduados em Educação da Universidade São Francisco, Bragança Paulista-SPP.

Perfetti, C. A. A. (1992). Capacidade para a leitura. Em R. J. Sternberg (Org.), As capacidades intelectuais humanas (pp. 72-76). Artes Médicas.

Santos, A. A. A. (1990a). Compreensão em leitura na universidade: um estudo comparativo entre dois procedimentos de treino. Estudos de Psicologia, 7 (2), 39-53.

Santos, A. A. A. (1990b). Leitura e universidade: uma análise de algumas questões críticas. Trans-informação, 2 (2-3), 91-104.

Santos, A. A. A. (1991). Desempenho em leitura: um estudo diagnóstico da compreensão e hábitos de leitura entre universitário. Estudos de Psicologia, 8 (1), 6-19.

Santos, A. A. A. (1997). Psicopedagogia no $3^{\circ}$ grau: avaliação de um programa de remediação em leitura e estudo. Pro-Posições, 8 (1), 27-37.

Santos, A. A. A.; Primi, R.; Taxa, F. \& Vendramini, C. M. M. (2002). O teste de Cloze na avaliação da compreensão em leitura. Psicologia: Reflexão $e$ Crítica, 15 (3), 549-560.
Santos, A. A. A.; Vendramini, C. M. M.; Suehiro, A. C. B. \& Santos, L. A. D. (2002). Compreensão de leitura em universitários: comparação entre ingressantes e concluintes de psicologia. Manuscrito submetido à publicação.

Storey, P. (1997). Examining the test-talking process: a cognitive perspective on the discourse Cloze test. Language testing, 14 (2), 214-231.

Veríssimo, L. F. (1995). Desentendimento. Ícaro Revista de bordo da Varig, 136.

Vicentelli, H. (1999). Problemática de la lectura en estudiantes universitarios. Psicologia Escolar e Educacional, 3 (3), 195-202.

Witter, G. P. (1997). Leitura e Universidade. Em G. P. Witter (Org.), Leitura e universidade (pp. 09-18). Campinas: Alínea.
Recebido: 26.06 .2003

Revisado:28.07.2003 Aceito:31.07.2003 


\section{Sobre os autores}

Katya Luciane de Oliveira: Psicóloga e aluna bolsista CAPES do Programa de Estudos Pós-Graduados em Psicologia, da Universidade São Francisco. Endereço para correspondência: Rua Maestro Sebastião Peranovich, 415 - Atibaia Jardim - Atibaia São Paulo - 12940-000 - fone: (11) 4411-9773 - e-mail: katya_lincoln@ig.com.br.

Acácia Aparecida Angeli dos Santos: Doutora em Psicologia pela USP, docente do curso de Psicologia e do Programa de Estudos Pós-graduados em Psicologia, da Universidade São Francisco.

Ricardo Primi: Doutor em Psicologia pela USP, docente do curso de Psicologia e do Programa de Estudos Pós-graduados em Psicologia, da Universidade São Francisco. 